\title{
Gastrointestinal stromal tumor of the colon - endoscopic treatment by full-thickness resection
}

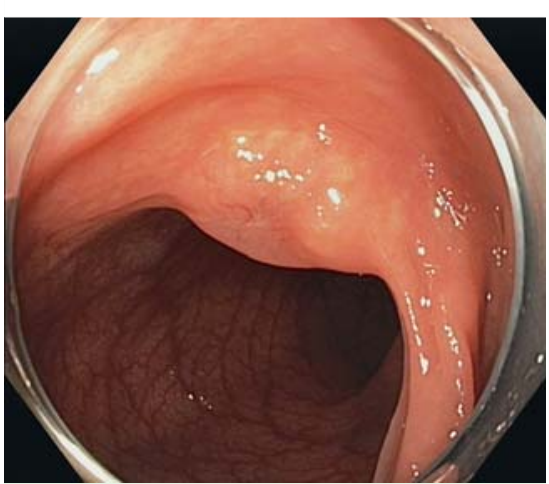

Fig. 1 Gastrointestinal stromal tumor of the sigmoid colon (superficial scar caused by previous biopsy) diagnosed during screening colonoscopy in a 51-year-old man.

A 51-year-old man was referred for therapy of a submucosal tumor of the sigmoid colon. The lesion had been diagnosed during screening colonoscopy, and biopsies had shown gastrointestinal stromal tumor (GIST). We confirmed a $15-\mathrm{mm}$ submucosal tumor located in the proximal sigmoid colon ( $\bullet$ Fig. 1 ).

Endoscopic full-thickness resection was performed with the Full Thickness Resection Device (FTRD; Ovesco Endoscopy, Tübingen, Germany) ( $\bullet$ Fig. 2, $\bullet$ Video 1 ). The FTRD system and first clinical experience were recently described by Schmidt et al. [1]. The resection was performed while the patient was under conscious sedation with midazolam and propofol. The procedure time was 25 minutes, and the remainder of the course was uneventful. Histologic analysis of the $30-\mathrm{mm}$ specimen confirmed full-thickness resection of the colonic wall, which contained a 12-mm GIST originating from the muscularis propria layer ( $\bullet$ Fig.3). The resected GIST specimen was R0 and showed 2 mitoses $/ 5 \mathrm{~mm}^{2}$. The resection was judged to be curative, and the patient was followed without further treatment.

GISTs of the colon are rare. In a large study of 4411 GISTs, the stomach was the predominant location (60.3\%); colonic lesions accounted for only $2.9 \%$ and rectal lesions for another $3.1 \%$ [2]. For gastric GISTs, several endoscopic resection techniques have been reported, including full-thickness resection with or without laparoscopic hybrid techniques [3]. For colonic GISTs originating from the muscularis propria layer, surgical resection has usually been needed to date to achieve complete resection without perforation [4].

After the introduction of the over-thescope clip (OTSC; Ovesco Endoscopy), endoscopic full-thickness resection in the colorectum became possible. A "pseudopolyp" containing the complete colonic wall is created with application of the OTSC, and snare resection of the pseudo- polyp is performed afterward [5]. The novel FTRD system allows endoscopic full-thickness resection of colorectal lesions in one step. Endoscopic resection with the FTRD system should be considered the treatment of choice for small colorectal submucosal tumors when resection is needed (e.g., when GIST is confirmed or when the histology is unknown).

Endoscopy_UCTN_Code_TTT_1AQ_2AD

\section{Competing interests: None}

\section{Andreas Probst ${ }^{1}$, Tina Schaller ${ }^{2}$, Helmut Messmann'}

${ }^{1}$ Department of Gastroenterology, Klinikum Augsburg, Germany

${ }^{2}$ Institute of Pathology, Klinikum Augsburg, Germany

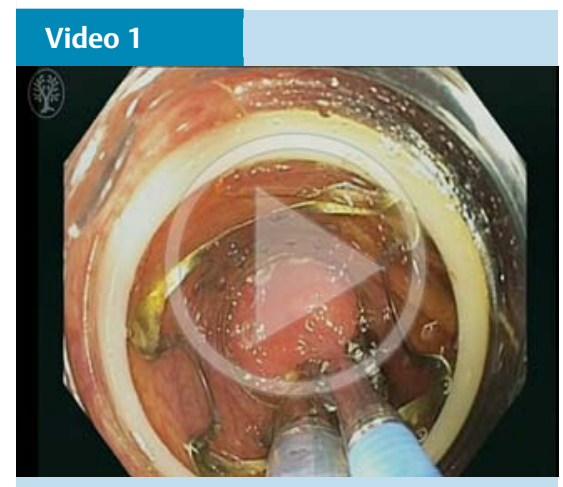

Endoscopic full-thickness resection of a gastrointestinal stromal tumor of the sigmoid colon with the Full Thickness Resection Device.
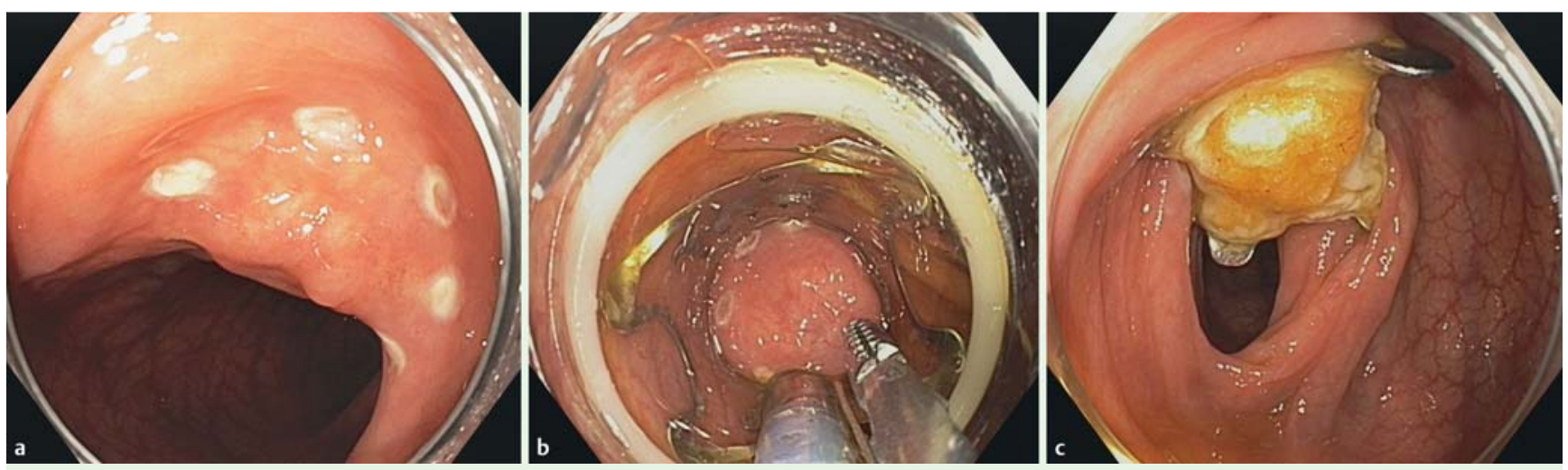

Fig. 2 Endoscopic full-thickness resection of the colonic gastrointestinal stromal tumor with the Full Thickness Resection Device (FTRD) system. a Coagulation marks are applied to the lesion. $\mathbf{b}$ The lesion is grasped into the transparent cap of the FTRD. Coagulation marks can be seen at the lateral margin, and the over-the-scope clip (OTSC) is visible on the transparent cap. c Endoscopic view after the procedure shows full-thickness resection closed with an OTSC. 


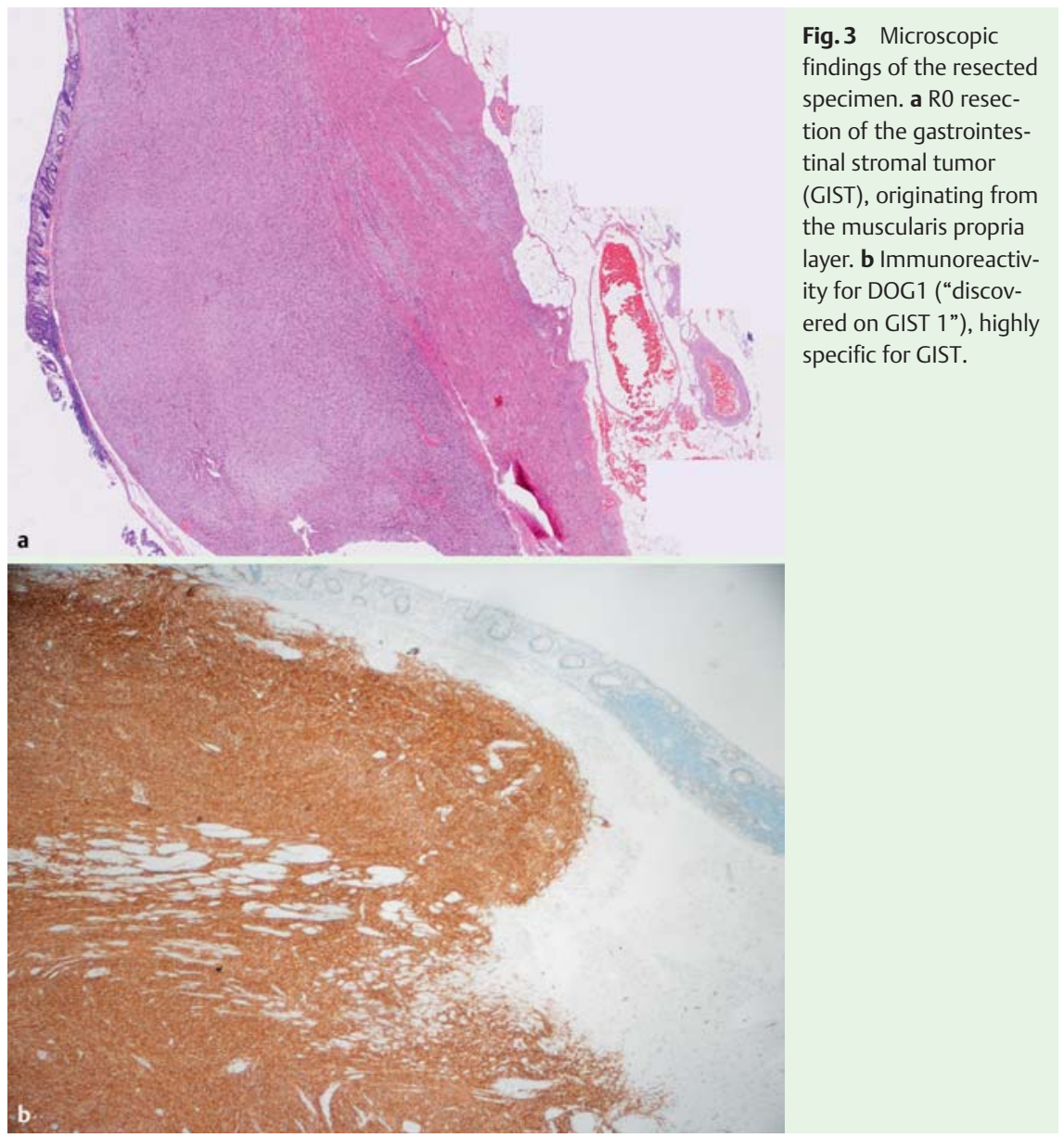

\section{References}

1 Schmidt A, Bauerfeind P, Gubler C et al. Endoscopic full-thickness resection in the colorectum with a novel over-the-scope device: first experience. Endoscopy 2015; 47: 719 725. DOI: 10.1055/s-0034-1391781. Epub 2015 Mar 12

2 Kukar M, Kapil A, Papenfuss W et al. Gastrointestinal stromal tumors (GISTs) at uncommon locations: a large population based analysis. J Surg Oncol 2015; 111: 696- 701

$3 \mathrm{Kim} \mathrm{HH}$. Endoscopic treatment for gastrointestinal stromal tumor: advantages and hurdles. World J Gastrointest Endosc 2015; 16: $192-205$

4 Chen CW, Wu CC, Hsiao CW et al. Surgical management and clinical outcome of gastrointestinal stromal tumor of the colon and rectum. Z Gastroenterol 2008; 46: $760-765$

5 Fähndrich $M$, Heike M, Sandmann M. The Dortmund endoscopic full-thickness resection method: combination of the over-thescope clip and the Inoue cap. Endoscopy 2014; 46 (Suppl. 01): E466. DOI: 10.1055/s0034-1377548. Epub 2014 Oct 14

\section{Bibliography}

DOI http://dx.doi.org/

10.1055/s-0034-1392862

Endoscopy 2015; 47: E460-E461

(c) Georg Thieme Verlag KG

Stuttgart · New York

ISSN 0013-726X

\section{Corresponding author}

\section{Probst, MD}

III. Medizinische Klinik

Klinikum Augsburg

Stenglinstrasse 2

86156 Augsburg

Germany

Fax: +49-821-400-3331

andreas.probst@klinikum-augsburg.de 